Vol. 01, No. 04, octubre - diciembre 2021, 30-42

\title{
Caracterización de un jardín clonal de cacao (Theobroma cacao L.) en la amazonía peruana
}

\section{Characterization of a clonal cocoa garden (Theobroma cacao L.) in the peruvian amazon}

\author{
Máximo Parco ${ }^{1, a, *}$, Alina A. Camacho $2, b$, Jhimy A. Parco ${ }^{3, c}$, Fiorela E. Dionisio ${ }^{4, d}$ \\ ${ }^{1}$ Instituto Nacional de Innovación Agraria, Junín, Perú. \\ ${ }^{2}$ Instituto Nacional de Innovación Agraria, Pucallpa, Perú. \\ ${ }^{3}$ Universidad Nacional Hermilio Valdizan, Huánuco, Perú. \\ ${ }^{4}$ Universidad Nacional del Centro del Perú, Huancayo, Perú. \\ ${ }^{a}$ Ing., $\bigotimes_{\text {mparcoq@gmail.com, (D) https://orcid.org/0000-0002-0040-4086 }}$

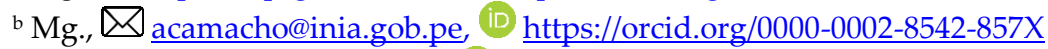 \\ cIng., ihmparco@gmail.com, (D) https://orcid.org/0000-0001-7958-3197 \\ ${ }^{\mathrm{d} B a c h .,} \unrhd_{\text {fiodisa2711@gmail.com, }}$ (D) https://orcid.org/0000-0002-9769-6782 \\ * Autor de Correspondencia: Tel. +51966569918 \\ http://dx.doi.org/10.25127/riagrop.20214.719
}

http://revistas.untrm.edu.pe/index.php/RIAGROP

revista.riagrop@untrm.edu.pe

Recepción: 02 de agosto 2021

Aprobación: 25 de agosto 2021

Este trabajo tiene licencia de Creative Commons. Attribution-NonCommercial-ShareAlike $\quad 4.0$ International Public License - CC-BY-NC-SA 4.0

\section{Resumen}

La variabilidad genética de clones de cacao (Theobroma cacao L.), que existe en el jardín clonal de la Estación Experimental Pichanaqui, de procedencia nacional e internacional, plantea la necesidad de realizar una caracterización morfológica a nivel de frutos, con descriptores morfológicos y escalas para cada tipo de tejido colectado, que usaron como auxiliares las siglas, los colores y formas que permitieron diferenciar las características de cada individuo. Las variables evaluadas fueron número de frutos por planta, peso de mazorcas maduras, numero de granos por mazorca, peso de almendras seca por mazorca, peso de 10 semillas secas, presencia de plagas como la Moniliasis (Moniliophthora roreri), Escoba de bruja (Moniliophthora perniciosa), Mancha parda (Phytophthora palmivora), Perforador (Carmenta foraseminis), Chinche amarillo (Monalonium dissimulatum) y calidad organoléptica en pulpa fresca. Además, se evaluaron variables económicas como el ingreso neto del clon por hectárea. Los resultados indicaron que los mejores clones 
por su productividad, tolerancia media a plagas y enfermedades, rentabilidad y sabor fueron: ICS 1, ICS 60, SCA 6, ICS 39, y TSH 565. En conclusión, se debería fomentar la siembra de estos clones en los productores de la zona a fin de mejorar su competitividad en el mercado local y nacional.

Palabras claves: Selección, productividad, morfología, clon, germoplasma.

\begin{abstract}
The genetic variability of cocoa clones (Theobroma cacao L.) that exist in the clonal garden of the Pichanaqui Experimental Station, of national and international origin, raises the need to carry out a morphological characterization at the fruit level, with morphological descriptors and scales for each type of tissue collected, using as auxiliary acronyms, colors and shapes that allow differentiating the characteristics of each individual. The variables evaluated were number of fruits per plant, weight of ripe ears, number of grains per ear, weight of dry almonds per ear, weight of 10 dry seeds, presence of pests such as Moniliasis (Moniliophthora roreri), witch's broom (Moniliophthora perniciosa), Brown spot (Phytophthora palmivora), Perforator (Carmenta foraseminis), Yellow bug (Monalonium dissimulatum) and organoleptic quality in fresh pulp. In addition, economic variables such as the clone's net income per hectare were evaluated. The results indicated that the best clones for their productivity, medium tolerance to pests and diseases, profitability and flavor were: ICS 1, ICS 60, SCA 6, ICS 39, and TSH 565; In conclusion, the sowing of these clones should be encouraged in the producers of the area in order to improve their competitiveness in the local and national market.
\end{abstract}

Keywords: Selection, productivity, morphology, clone, germplasm.

\section{INTRODUCCIÓN}

El cacao tiene significativa relevancia social por ser el sexto cultivo más importante a nivel mundial y en América Latina y Central, ocupa el $14.9 \%$ de la producción en grano (Gorotiza et al., 2020). Más de 138000 productores tienen cacao instalado en sus unidades productoras agropecuarias, en la selva peruana. De todos ellos, más de 111000 productores cosecharon cacao en la campaña 2015 a 2016, según los últimos datos disponibles (Ministerio de Agricultura y Riego, 2018).

La caracterización morfológica del cacao, tanto del fruto como características de las semillas, es importante porque permite establecer parámetros que identificaran al cultivar $\mathrm{y}$ ayudarán a facilitar la diferenciación o la comparación con otros cultivares que puedan estar presentes en la zona (Food and Agriculture Organization, 2002; Prado, 2019). El jardín clonal de cacao de la Estación Experimental Pichanaki resulta importante en su caracterización morfológica ya que está compuesto por clones nacionales e introducidos de otros países que nos permiten identificar, conocer su comportamiento de adaptación, productividad y tolerancia a las principales plagas y seleccionar clones élites de superior productividad.

En México, se han descrito morfológicamente accesiones con forma de fruto calabacillo, amelonado, angoleta y cundeamor por parte del Instituto Nacional de Investigaciones Forestales, Agrícolas y Pecuarias (INIFAP), que implican diferencias en calidad del grano entre las mismas (Ramírez-Guillermo et al., 2018). 
Oliva (2020), en su trabajo de caracterización de la diversidad biológica de cacao, estableció cinco grupos claramente diferenciados entre sí, que presentan las mejores características sensoriales y de rendimiento.

Actualmente, los productores de la región Junín no utilizan el potencial material genético existente, por desconocimiento y continúan cultivando los materiales genéticos de baja productividad. El jardín clonal de la Estación Experimental Pichanaki en estudio comprende de 16 clones instalados en el año 2010, en el cual se encuentran materiales genéticos valiosos. Por ello, la presente investigación tiene como objetivo realizar la caracterización de un jardín clonal de cacao (Theobroma cacao L.) en la amazonia peruana.

\section{MATERIALES Y MÉTODOS}

\subsection{Localización}

La investigación se realizó en el jardín clonal de la Estación Experimental Agraria Pichanaki, ubicado a los $540 \mathrm{~m} \mathrm{s.} \mathrm{n.} \mathrm{m.} \mathrm{con} \mathrm{una} \mathrm{temperatura}$ promedio anual $24.9{ }^{\circ} \mathrm{C}$ y con una precipitación promedio anual de $1500 \mathrm{~mm}$ (SENAMHI, 2019), en el distrito de Pichanaqui, provincia de Chanchamayo, región Junín (figura 1).

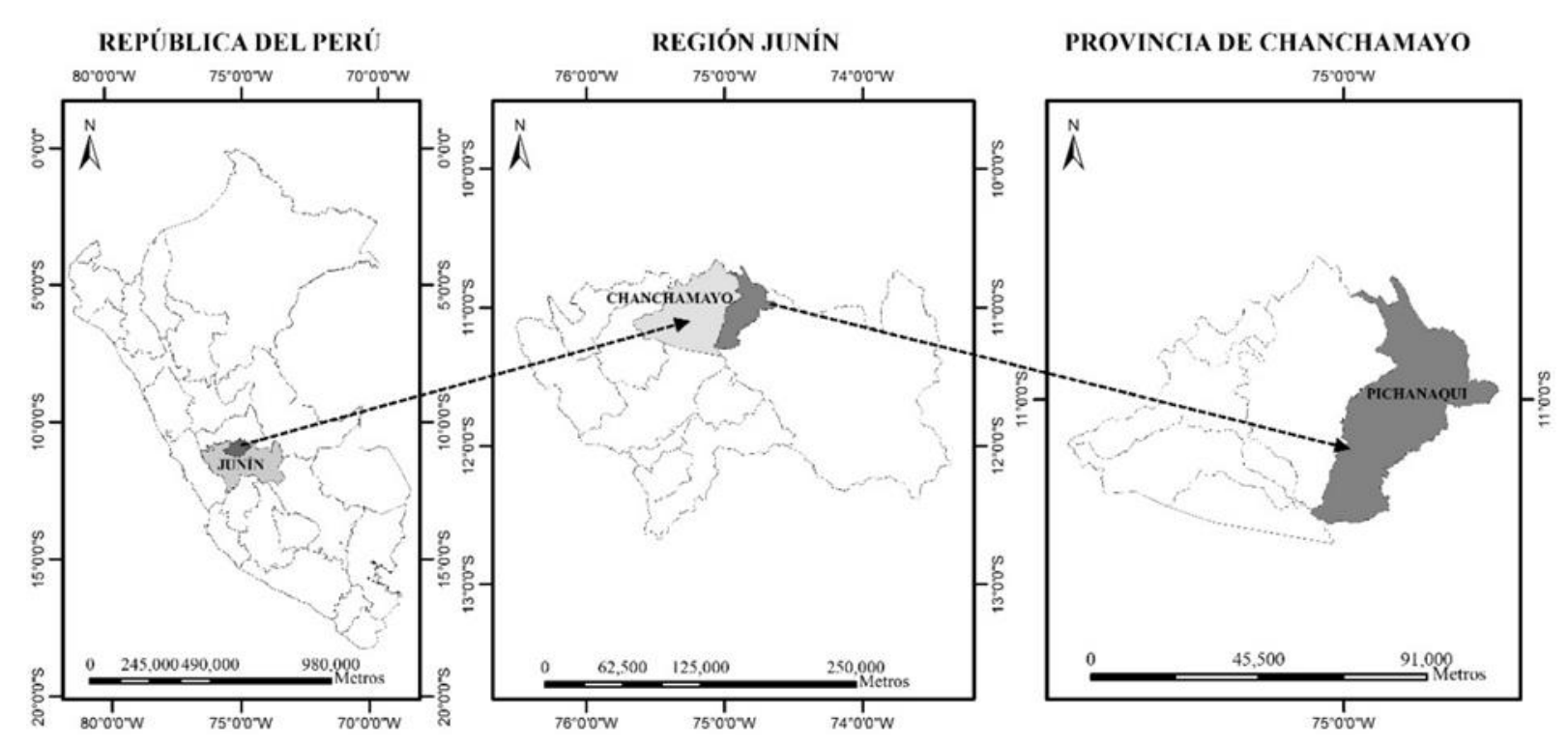

Figura 1. Ubicación del área de estudio.

\subsection{Material biológico}

Los materiales utilizados fueron los 16 clones que se encuentran instalados en el jardín clonal de la Estación Experimental Agraria Pichanaki. Los clones fueron: ICS.6, ICS-1, IMC 67, ICS-60, SCA-6, ICS-39, TSH 565, EET-228, H58, IAC-1， CCN-51， H-46, U-10, ICS-95, Porcelana y Rosario, que se instalaron con un distanciamiento de siembra de $3 \mathrm{~m} \times 3 \mathrm{~m}$, en hileras de 10 plantas de cada clon, de los cuales se evaluaron cuatro plantas centrales.

\subsection{Metodología}

El experimento se realizó con la relación de clones de cacao mencionados en jardín clonal sin diseño experimental. La evaluación se 
realizó en campo y laboratorio. En la etapa de maduración de frutos, se realizó la caracterización morfológica de frutos con descriptores morfológicos según la guía de Garcia (2010), se utilizó descriptores de flores, frutos, y semillas.

\subsubsection{Descriptores de fruto $(N=10)$}

\section{Color del fruto inmaduro (CFI)}

Se evaluó visualmente, considerando los siguientes estados:

$0=$ verde, 2 = verde pigmentado, $3=$ rojo.

\section{Forma del fruto (FOF)}

Se determinó de acuerdo a los siguientes estados:

1 = oblongo, 2 = elíptico, 3 = abovado,

4 = esférico, $5=$ otros.

\section{Forma del ápice del fruto (FAF)}

Se determinó de acuerdo a los siguientes estados:

$1=$ atenuado, 2 = agudo, 3 = obtuso,

$4=$ redondeado, 5 = apezonado, $6=$ dentado.

\section{Rugosidad de la superficie del fruto (RUF)}

Se evaluó la presencia o ausencia de protuberancias en la superficie del fruto, que incluye los siguientes estados: $0=$ ausente, $3=$ ligero, 5 = intermedio, $7=$ fuerte .

\section{Constricción basal del fruto (CBF)}

Se determinó según el grado de depresión o cuello de botella de la base del fruto. Los estados del descriptor se detallan continuación:

0 = ausente, 3 = ligera, 5 = intermedia, $7=$ fuerte.

\section{Profundidad de los surcos primarios (PSP)}

Se evaluó considerando los siguientes estados: $3=$ superficial, 5 = intermedio, $7=$ profundo.

\section{Separación de un par de lomos (SPL)}

Se realizó en forma visual considerando los siguientes estados:

$0=$ fusionados, 3 = ligera, 5 = intermedia,

$7=$ separados.

\section{Grosor de cáscara (GC)}

Se evaluó teniendo en cuenta lo siguiente: $1=$ delgada, $2=$ intermedia y 3 = gruesa.

\subsubsection{Descriptores de semilla}

Forma de la semilla en sección longitudinal (FSL)

Se determinó tomando en cuenta los siguientes estados:

1 = oblonga, 3 = elíptica, 5 = ovada, 7 = irregular .

\section{Forma de la semilla en sección transversal (FST)}

Se evaluó considerando los siguientes estados: 1 = aplanada, 3 = intermedia, 5 = redondeada.

\section{Sabores básicos de la pulpa fresca}

La evaluación los sabores básicos de la pulpa fresca y almendra, se realizó utilizando la escala del $0-5$ en donde: $0=$ nulo, $1=$ muy bajo, 2 = bajo, 3 = medio, 4 = alto (fuerte), $5=$ muy alto, muy fuerte.

\subsubsection{Evaluación sanitaria}

Para la evaluación de reacción a la presión de las principales enfermedades que son: la Moniliasis (Moniliophthora roreri), Escoba de bruja (Monilophthora perniciosa) y Mancha Parda (Phytophthora palmívora) se utilizaron la escala siguiente: $\mathrm{R}=$ resistente, $\mathrm{MR}=$ moderadamente resistente, $\mathrm{T}=$ tolerante, $\mathrm{MS}=$ moderadamente susceptible, $\mathrm{S}=$ Susceptible. 


\subsubsection{Variables de productividad}

Se evaluó el número de frutos por planta, peso de mazorcas maduras (g), diámetro y mazorcas $(\mathrm{cm})$, longitud de mazorca $(\mathrm{cm})$, espesor de lomo $(\mathrm{cm})$, ranura $(\mathrm{cm})$, número de granos por mazorca, peso de grano con mucílago, peso de almendra seca por mazorca, por planta y por hectárea, peso de una almendra seca y peso de 10 almendras secas, utilizando la balanza de precisión.

\subsubsection{Incidencia de plagas y enfermedades}

La evaluación de incidencia de ataque de las principales plagas se realizó mensualmente en el campo, las principales plagas evaluadas fueron: la Moniliasis (Moniliophthora roreri) en frutos; Escoba de bruja (Moniliophthora perniciosa) en cojines florales, en frutos y brotes terminales; Mancha parda (Phytophthora palmivora) en frutos; Mazorquero (Carmenta foraseminis) en frutos; Chinchi amarillo (Monalonium dissimulatum) en frutos y Asta de torito (Hoplophorium pertusa) en frutos, que son plagas potenciales que se encuentran en toda las parcelas cacaoteras de la Selva Central. Se evaluaron los daños de estas plagas mediante la cuantificación individual de la presencia de síntomas de ataque de cada plaga en frutos y tallos que se presenta, de acuerdo a la susceptibilidad de cada clon de cacao y factores ambientales favorables.

\subsubsection{Variables económicas}

Se consideró las variables ingreso bruto de clon por hectárea, gastos de mantenimiento y el ingreso neto de clon por hectárea.

\subsection{Diseño experimental y análisis de datos}

Los datos de productividad e incidencia de plagas que se presentan en tablas, cuadros, $y$ gráficos son promedios de 5 años de evaluación de los clones desde 2014 hasta 2018.

\section{RESULTADOS Y DISCUSIÓN}

\subsection{Optimización de múltiples variables con la metodología superficie de respuesta (MSR) para las biopelículas antimicrobianas}

De los 16 clones de cacao, 8 pertenecen al grupo genético de los forasteros, 1 cacao híbrido (Ecuador), Híbrido (Trinidad y Tobago), 1 nacional y 5 trinitarios. Con respecto al color del fruto inmaduro (CFI), 4 son rojos, 11 verdes $y$ solo 1 verde pigmentado. La mayor cantidad se hace presente con 11 verdes. Según Ballesteros et al. (2016), este color de fruto inmaduro es una de las principales características que ayuda en su caracterización morfológica.

De los 16 evaluados, 6 son elípticos y 10 oblongos. Para la forma del ápice agudo (FAF), se encontraron 6 agudos, 1 apezonado, 6 atenuados y 3 obtusos. Para rugosidad de la superficie del fruto se encontró que 1 fruto de los 16 clones presenta una rugosidad fuerte, 8 están se clasifican como intermedios y 7 ligeros (tabla 1). El presente estudio permitió caracterizar ecotipos de acuerdo a los descriptores morfológicos los cuales ayudan en la diferenciación fenotípica de clones de cacao (García, 2020).

En la tabla 2, se puede observar que constricción basal del fruto (CBF), 4 frutos son intermedios y 12 ligeros, en la caracterización realizada por Ramírez-Guillermo et al. (2018) reportaron en una población de 101 clones, para esta variable 
36 ausentes, 35 escasos, 16 intermedios y 19 marcados. La característica de intermedios reportó el valor mínimo y ausente el valor máximo. Estos resultados fueron similares a los reportados en esta investigación, donde los promedios más altos se encuentran en la característica ligera y un bajo reporte para intermedios. Para grosor de la cáscara (GC), 3 son delegadas, 1 gruesa y 12 intermedias. Con respecto a una separación de un par de lomos
(SPL), encontramos que 13 son intermedios y 3 ligeros. Para la profundidad de los surcos primarios (PSP) encontramos a 11 intermedios y 3 ligeros, 1 profundo y 1 superficial. Para el caso de descriptores de semilla en la variable evaluada forma de la semilla en sección longitudinal (FSL) encontramos 12 elípticas y 4 oblongas. Finalmente, para forma de semilla en sección transversal (FST), 3 se caracterizan por ser aplanadas y 13 intermedias.

Tabla 1. Caracterización morfológica de frutos del jardín clonal de cacao

\begin{tabular}{|c|c|c|c|c|c|}
\hline CLON & $\begin{array}{l}\text { GRUPO } \\
\text { GENÉTICO }\end{array}$ & CFI & FOF & FAF & RUF \\
\hline ICS-6 & Trinitario & verde & elíptica & apezonado & ligera \\
\hline ICS-1 & Trinitario & rojo & elíptica & obtuso & ligera \\
\hline IMC- 67 & Forastero & verde & oblongo & atenuado & ligera \\
\hline ICS -60 & Trinitario & verde & elíptica & agudo & intermedia \\
\hline SCA- 6 & Forastero & verde & oblongo & atenuado & ligera \\
\hline ICS -39 & Trinitario & $\begin{array}{l}\text { verde } \\
\text { pigmentado }\end{array}$ & oblongo & agudo & intermedia \\
\hline TSH -565 & $\begin{array}{l}\text { Hibrido (Trinidad y } \\
\text { Tobago) }\end{array}$ & rojo & oblongo & obtuso & intermedia \\
\hline EET -228 & Nacional & verde & elíptica & atenuado & intermedia \\
\hline $\mathrm{H}-58$ & Forastero & Verde & elíptica & agudo & intermedia \\
\hline IAC- 1 & Forastero & Verde & elíptica & agudo & intermedia \\
\hline CCN- 51 & Híbrido (Ecuador) & Rojo & oblongo & atenuado & fuerte \\
\hline $\mathrm{H}-46$ & Forastero & Verde & oblongo & obtuso & ligera \\
\hline $\mathrm{U}-10$ & Forastero & Verde & oblongo & atenuado & ligera \\
\hline ICS- 95 & Trinitario & Rojo & oblongo & agudo & intermedia \\
\hline Porcelana & Forastero & Verde & oblongo & atenuado & ligera \\
\hline ROSARIO & Forastero & Verde & oblongo & agudo & intermedia \\
\hline
\end{tabular}

CFI: Color de fruto inmaduro; FOF: Forma del fruto; FAF: Forma del ápice del fruto; RUF: Rugosidad de superficie del fruto. 
Tabla 2. Caracterización morfológica de frutos y semillas del jardín clonal de cacao

\begin{tabular}{lllllll}
\hline CÓDIGO & CBF & GC & SPL & PSP & FSL & FST \\
\hline ICS-6 & ligera & intermedia & Intermedia & intermedia & elíptica & aplanada \\
ICS-1 & ligera & intermedia & Intermedia & intermedia & elíptica & intermedia \\
IMC- 67 & ligera & gruesa & Intermedia & superficial & oblonga & aplanada \\
ICS -60 & intermedia & intermedia & Intermedia & intermedia & elíptica & intermedia \\
SCA- 6 & intermedia & delgada & Ligera & ligera & oblonga & aplanada \\
ICS -39 & intermedia & delgada & Ligera & intermedia & oblonga & intermedia \\
TSH -565 & ligera & intermedia & Ligera & intermedia & elíptica & intermedia \\
EET -228 & intermedia & intermedia & Intermedia & intermedia & elíptica & intermedia \\
H -58 & ligera & intermedia & Intermedia & intermedia & elíptica & intermedia \\
IAC- 1 & ligera & intermedia & Intermedia & intermedia & elíptica & intermedia \\
CCN- 51 & ligera & intermedia & Intermedia & profundo & elíptica & intermedia \\
H - 46 & ligera & intermedia & Intermedia & ligera & elíptica & intermedia \\
U -10 & ligera & intermedia & Intermedia & ligera & oblonga & intermedia \\
ICS- 95 & ligera & delgada & Intermedia & intermedia & elíptica & intermedia \\
Porcelana & ligera & intermedia & Intermedia & intermedia & elíptica & intermedia \\
ROSARIO & ligera & intermedia & Intermedia & intermedia & elíptica & intermedia \\
\hline
\end{tabular}

CBF: Constricción Basal del fruto; GC: grosor de cáscara; SPL: Separación de un par de lomos; PSP: Profundidad de los surcos primarios; FSL: forma de semilla en sección longitudinal; FST: forma de semilla en sección transversal.

De los 16 clones evaluados en sabor básico, se encontraron 8 clones con sabor básico a frutal (ICS-6, IMC-67, SCA-6, ICS-39, H-58, IAC-1, U10 y Porcelana) y 8 con sabor básico a floral (ICS-1, ICS-60, TSH-565, EET-228, CCN-51, H46, ICS-95 y Rosario). La presencia de aromas es una característica del cacao fino, como lo menciona Xiao-Wei et al. (2017). Estos atributos contribuyen en la diferenciación comercialmente de los tipos de cacao con fines de exportación los mismos que se complementan con sus perfiles sensoriales (Álvarez et al., 2018).

En la figura 2a, se puede observar el número de frutos por planta obtenidos por cada clon evaluado. El clon con mayor número de frutos obtenidos fue el ICS-95, con 33 frutos por planta, que demuestra ser un clon de alta productividad, como lo mencionan Quintana et al. (2015), que corresponde a un cacao híbrido trinitario de alta productividad moderadamente resistente a enfermedades. Con respecto al peso de mazorcas, el clon ICS-6 reportó $825 \mathrm{~g}$ y el clon con un el peso más bajo fue el U-10 con 452 g (figura 2b). El mayor reporte alcanzado para diámetro de mazorcas fue alcanzado por el clon denominado Rosario, con $23 \mathrm{~cm}$ y el clon U-10 reportó el menor valor con $15 \mathrm{~cm}$ (figura 2c). Para el caso de longitud de mazorcas el clon ICS-6 alcanzó $34.3 \mathrm{~cm}$ y el clon ICS-95 alcanzó $21 \mathrm{~cm}$ (figura 2d). En el caso de espesor de cáscara referido a lomo, medido en cm, el clon ICS-60 reportó el valor más alto con $2.2 \mathrm{~cm}$ y el clon Porcelana reportó el valor más bajo con $1.3 \mathrm{~cm}$ (figura 2e). Para ranura el clon ICS-60, se registró el valor más alto con 1.7 cm y THS-565 reportó el valor más bajo con 0.8 cm (figura 2f). 


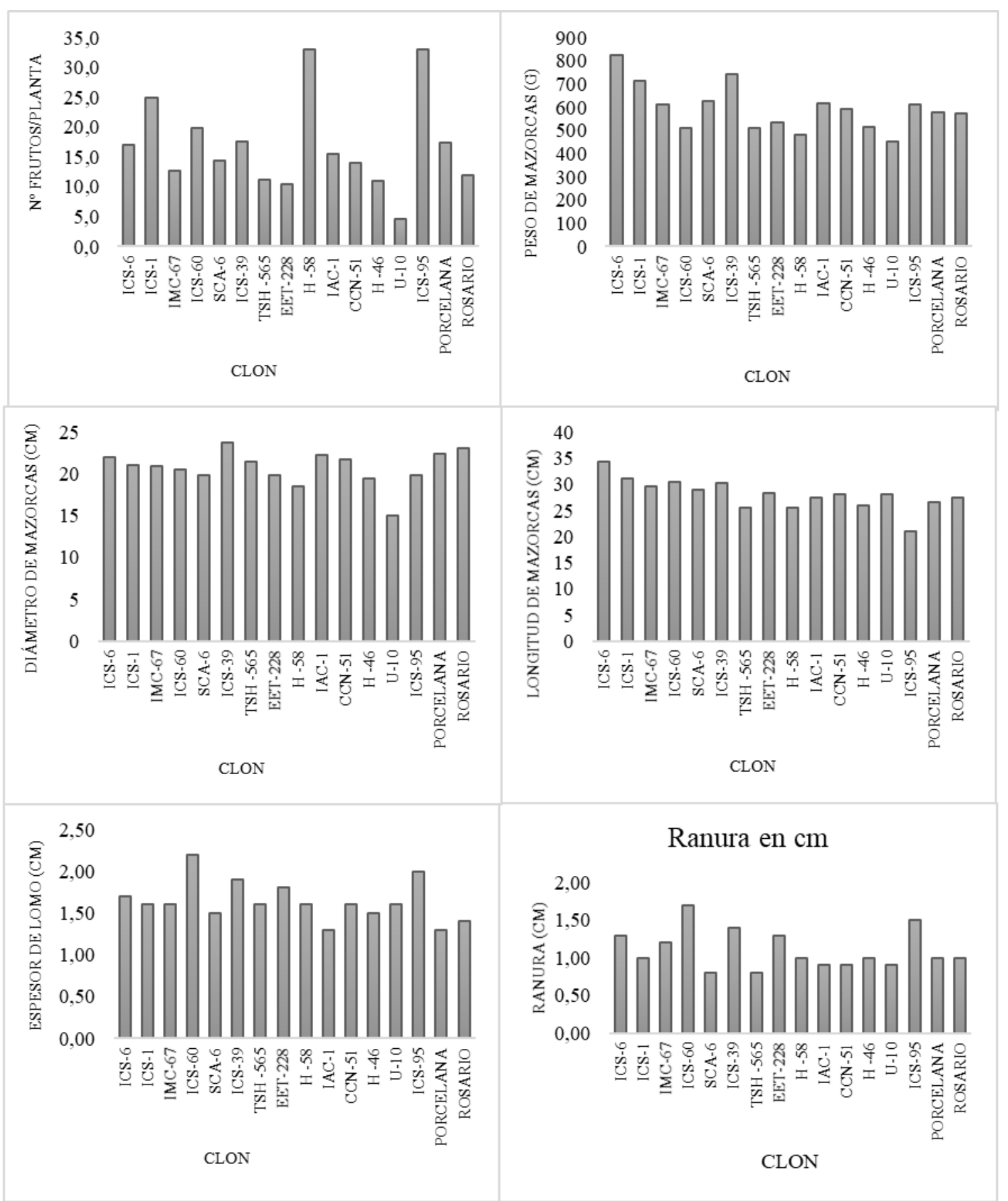

Figura 2. Evaluación de frutos, a: número de frutos por planta, b: peso de mazorca, c: diámetro de mazorcas, d: longitud de mazorcas, e: espesor de lomo y f: ranura del clon.

En la tabla 3, se puede observar para el número de granos por mazorca ocupa el primer lugar el Clon H-46 con 54.8 almendras, seguido por el clon SCA-6 con 53.1 almendras. En peso húmedo de almendras por mazorca, supera al resto el clon SCA-6 con 182.1 g, seguido por ICS-
6 con $182 \mathrm{~g}$ por mazorca. En peso seco de almendra por mazorca, sobresale el clon ICS-39 con 77.4 g por mazorca seguido por SCA-6 con $75 \mathrm{~g}$. En peso de 10 almendras secas, el clon ICS39 sobresale con $19.00 \mathrm{~g}$, seguido por los clones ICS-60 CON 18.9 y EET-228, con $18 \mathrm{~g}$. 
Finalmente para peso de una almendra seca, sobresalen los clones ICS-60 y ICS-39, ambos con $1.9 \mathrm{~g}$. El peso seco de semilla registrado por Quiñones et al. (2018) fueron inferiores a lo encontrado en el presente trabajo de investigación con reportes de 1.25 a 2 g para clones de cacao evaluados en Piura. Esto demuestra que la zona presenta un buen potencial de productividad, ya que en el presente trabajo de investigación se presentan datos superiores a $1.25 \mathrm{~g}$. La caracterización del germoplasma, referido a la producción de granos por mazorca resulta importante para determinar, de esa manera, el índice de productividad de los clones de cacao (Vera et al., 2014).

Según evaluación en peso de almendra seca por planta, son atributos físicos de mucha importancia (Álvarez et al., 2018), los resultados reportados en la presente investigación. El clon ICS-1 con $1809.9 \mathrm{~g}$, seguido por el clon ICS - 60 con 1370.7 y $\quad$ ICS-39 con 1362.2 g respectivamente superan al resto de clones. En rendimiento de almendra seca por ha, supera al resto el clon ICS-1 con $2010.8 \mathrm{Kg} / \mathrm{ha}$, seguido por el clon ICS-60 con $1522.8 \mathrm{Kg}$ y clon ICS-39 con $1513.4 \mathrm{Kg}$. Estos atributos cambiantes podrían estar ligado al acondicionamiento genético y ambiental. En ese sentido, la etapa de la fecundación de la flor fue la determinante para el rendimiento y peso promedio de la almendra influida por la época estacional (Álvarez, 2018). El ingreso bruto es el valor de la venta total de almendras secas por ha, sin restar los costos de producción por campaña. Si se considera la suma de S/ 3000 igual para todos los tratamientos con una densidad de 1111 plantas por ha, el precio promedio de almendra seca fue la suma de $\mathrm{S} / 5.5 / \mathrm{kg}$. En evaluación de ingreso neto / ha, ocupa el primer lugar el clon
ICS-1 con S/ 8059.5/ha, seguido por el clon ICS60 con S/ 5375.4 y ICS-39 en S/ 5323.92 (tabla 4). En la tabla 5, de evaluación de incidencia de plagas, no se encontró síntomas de ataque en frutos por Perforador (Carmenta foraseminis), a excepción en el clon Porcelana con 0.3. Tampoco se encontró presencia de individuos de Chinche amarillo (M. dissimulatum) en frutos. Solamente, se encontró síntomas de daños en frutos en los clones, donde el mayor número de frutos infectados por planta se hace presente en los clones ICS-6 con 3.5 frutos atacados y ICS-1 con 3.3 frutos dañados y en los clones restantes el daño fue muy bajo.

En cuanto ataque de Moniliasis ( $M$. roreri) en frutos, fue escaso. Solo se observó ligeros daños en frutos de los clones EET - 228 y H-58, con un promedio de 0.5 . No se observó daños en los clones como ICS 95. Esto confirma la resistencia del material a Monilia, como lo afirma Quintana et al. (2018), quienes agrupan como material resistente a FEC 2, CAUC 3, ICS 95, FLE 2. En evaluación de daños de Escoba de bruja ( $M$. perniciosa), solo se observó muy leve daño en frutos del clon IMC - 67 con 0.3. En cambio, en cojines florales, hubo mayor presencia en los clones ICS - 60 con 1.5 y en ICS - 39 con 1.3. En brotes terminales, se observó daños en los clones ICS-39 con 1, EET - 228 y ICS-60 con 0.8 brotes dañados. En la evaluación de Mancha parda (P. palmivora) que ataca a todos los órganos de la planta de cacao, se encontró mayor ataque en los clones: IAC-1 con 3.3, seguido por el clon $\mathrm{H}-58$ con 3 y CCN - 51 con 2.5 frutos dañados. En la evaluación de la plaga Asta de torito (Hoplophorium pertusa), que ocasiona daños severos en pequeños frutos no se presentó. La mortandad de frutos, por efectos fisiológicos se presentó con mayor frecuencia en los clones IAC-1 con 4.3, H-58 y CCN-51 con un 
promedio de 2 frutos muertos. En cuanto a perseverancia de floración y fructificación, sobresale los ICS-6 con $7.8 \%$ floración y Porcelana con $5.5 \%$ de floración.
Los clones evaluados en condiciones de manejo agronómico potencian sus rendimientos $\mathrm{y}$ disminución de ataque de plagas $y$ enfermedades (Tarqui, 2020).

Tabla 3. Evaluación de peso de almendras y mazorcas en gramos

\begin{tabular}{|c|c|c|c|c|c|}
\hline CLON & $\begin{array}{l}\mathrm{N}^{\circ} \text { grano/ } \\
\text { mazorcas }\end{array}$ & $\begin{array}{l}\text { Peso de } \\
\text { grano } \\
\text { C/mucílago } \\
\text { (g) }\end{array}$ & $\begin{array}{l}\text { Peso seco de } \\
\text { almendra por } \\
\text { mazorca (g) }\end{array}$ & $\begin{array}{l}\text { Peso de diez } \\
\text { almendras } \\
\text { secas }(\mathrm{g})\end{array}$ & $\begin{array}{l}\text { Peso de una } \\
\text { almendra } \\
\text { seca }(g)\end{array}$ \\
\hline ICS-6 & 37.0 & 182.0 & 70.0 & 16.8 & 1.7 \\
\hline ICS-1 & 40.5 & 163.5 & 72.6 & 13.3 & 1.3 \\
\hline IMC-67 & 46.1 & 133.1 & 56.3 & 13.5 & 1.4 \\
\hline ICS-60 & 35.0 & 159.1 & 69.4 & 18.9 & 1.9 \\
\hline SCA-6 & 53.1 & 182.1 & 75.0 & 14.4 & 1.4 \\
\hline ICS-39 & 38.4 & 178.8 & 77.4 & 19.0 & 1.9 \\
\hline TSH -565 & 36.5 & 129.5 & 48.3 & 13.3 & 1.3 \\
\hline EET-228 & 31.8 & 132.6 & 59.6 & 18.0 & 1.8 \\
\hline H-58 & 34.4 & 95.3 & 45.0 & 13.5 & 1.4 \\
\hline IAC-1 & 45.4 & 133.5 & 62.3 & 11.1 & 1.1 \\
\hline CCN-51 & 51.5 & 173.7 & 70.5 & 13.5 & 1.4 \\
\hline H -46 & 54.8 & 134.7 & 55.8 & 11.0 & 1.1 \\
\hline U-10 & 34.0 & 83.5 & 36.5 & 12.0 & 1.2 \\
\hline ICS-95 & 35.0 & 106.0 & 47.0 & 13.2 & 1.3 \\
\hline Porcelana & 43.1 & 165.1 & 60.1 & 15.0 & 1.5 \\
\hline Rosario & 45.1 & 165.4 & 64.8 & 15.8 & 1.6 \\
\hline
\end{tabular}


Tabla 4. Evaluación de número de frutos por planta, almendra seca por mazorca, por planta y por ha. Ingreso bruto e ingreso neto por haltratamiento

\begin{tabular}{|c|c|c|c|c|c|c|c|}
\hline Clones & $\begin{array}{l}\text { Número } \\
\text { de frutos/ } \\
\text { planta }\end{array}$ & $\begin{array}{l}\text { Peso de } \\
\text { almendra } \\
\text { seca/mazorca } \\
\text { (g) }\end{array}$ & $\begin{array}{l}\text { Peso de } \\
\text { almendra } \\
\text { seca/planta } \\
\text { (g) }\end{array}$ & $\begin{array}{l}\text { Peso de } \\
\text { almendra } \\
\text { seca/ha }(\mathbf{k g})\end{array}$ & $\begin{array}{l}\text { Ingreso } \\
\text { bruto } \\
\text { S/ clon/ha }\end{array}$ & $\begin{array}{l}\text { Gastos de } \\
\text { mantenimiento } \\
\text { (S/) }\end{array}$ & $\begin{array}{l}\text { Ingreso neto } \\
\text { S//ha }\end{array}$ \\
\hline ICS - 6 & 17.1 & 70.0 & 1195.6 & 1272.8 & 7000.2 & 3000.0 & 4000.2 \\
\hline ICS - 1 & 24.9 & 72.6 & 1809.9 & 2010.8 & 11059.5 & 3000.0 & 8059.5 \\
\hline IMC - 67 & 12.7 & 56.3 & 714.5 & 793.8 & 4365.6 & 3000.0 & 1365.6 \\
\hline ICS - 60 & 19.8 & 69.4 & 1370.7 & 1522.8 & 8375.4 & 3000.0 & 5375.4 \\
\hline SCA - 6 & 14.4 & 75.0 & 1081.5 & 1201.5 & 6608.5 & 3000.0 & 3608.5 \\
\hline ICS - 39 & 17.6 & 77.4 & 1362.2 & 1513.4 & 8323.9 & 3000.0 & 5323.9 \\
\hline TSH - 565 & 11.2 & 48.3 & 540.0 & 599.9 & 3299.6 & 3000.0 & 299.6 \\
\hline EET - 228 & 10.4 & 59.6 & 621.0 & 890.00 & 4894.8 & 3000.0 & 1894.8 \\
\hline $\mathrm{H}-58$ & 32.9 & 45.0 & 1481.4 & 1645.8 & 902.1 & 3000.0 & 6052.1 \\
\hline IAC - 1 & 15.6 & 62.3 & 970.6 & 1077.7 & 5977.2 & 3000.0 & 2927.2 \\
\hline $\mathrm{CCN}-51$ & 14.0 & 70.5 & 988.4 & 1098.1 & 6039.7 & 3000.0 & 3039.7 \\
\hline$H-46$ & 11.0 & 55.8 & 612.1 & 880.1 & 4840.4 & 3000.0 & 1840.4 \\
\hline$U-10$ & 4.7 & 36.5 & 170.5 & 189.8 & 1043.6 & 3000.0 & 1956.4 \\
\hline ICS - 95 & 12.0 & 47.0 & 564.0 & 600.6 & 3446.3 & 3000.0 & 446.0 \\
\hline Porcelana & 17.4 & 60.0 & 1045.2 & 1161.2 & 6386.7 & 3000.0 & 3386.7 \\
\hline Rosario & 11.9 & 64.8 & 767.9 & 853.1 & 4692.1 & 3000.0 & 1652.1 \\
\hline
\end{tabular}


Tabla 5. Evaluación de número de frutos infectados por plagas en jardín clonal de cacao

\begin{tabular}{|c|c|c|c|c|c|c|}
\hline \multirow{2}{*}{ Clon } & \multirow{2}{*}{$\begin{array}{l}\begin{array}{c}\text { (Monalonium } \\
\text { disimulatum) }\end{array} \\
\mathbf{N}^{\mathrm{o}} \text { de frutos } \\
\text { infectados/plata }\end{array}$} & \multicolumn{2}{|c|}{$\begin{array}{l}\text { Escoba de bruja } \\
\text { (Moniliophthora pernisiosa) }\end{array}$} & \multirow{2}{*}{$\begin{array}{l}\text { Mancha parda } \\
\text { (Phytophthora } \\
\text { palmivora) }\end{array}$} & \multirow{2}{*}{$\begin{array}{l}\text { Frutos } \\
\text { muertos }\end{array}$} & \multirow{2}{*}{$\begin{array}{l}\text { \% de floración y } \\
\text { fructificación }\end{array}$} \\
\hline & & $\begin{array}{l}\mathbf{N}^{\circ} \text { de cojines } \\
\text { florales } \\
\text { infestados }\end{array}$ & $\begin{array}{l}\mathrm{N}^{\circ} \text { de brotes } \\
\text { terminales }\end{array}$ & & & \\
\hline ICS-6 & 3.5 & 0.0 & 0.3 & 0.3 & 0.8 & 7.8 \\
\hline ICS-1 & 3.3 & 0.8 & 0.3 & 0.3 & 1.0 & 0 \\
\hline IMC-67 & 0.5 & 0.0 & 0.0 & 0.5 & 0.3 & 0.0 \\
\hline ICS-60 & 2.5 & 1.5 & 0.8 & 1.8 & 1.3 & 0.0 \\
\hline SC-6 & 2.0 & 0.5 & 0.0 & 0.8 & 1.3 & 0.0 \\
\hline ICS-39 & 0.8 & 1.3 & 1.0 & 0.8 & 0.8 & 0.0 \\
\hline TSH -565 & 0.5 & 0.5 & 0.5 & 0.3 & 1.3 & 0.0 \\
\hline EET-228 & 0.5 & 0.5 & 0.8 & 1.0 & 0.8 & 0.3 \\
\hline H-58 & 2.3 & 0.0 & 0.0 & 3.0 & 2.0 & 0.0 \\
\hline IAC-1 & 1.0 & 0.0 & 0.0 & 3.3 & 4.3 & 2.5 \\
\hline $\mathrm{CCN}-51$ & 0.0 & 0.0 & 0.0 & 2.5 & 2.0 & 0.0 \\
\hline H-46 & 0.5 & 0.5 & 0.0 & 0.5 & 0.0 & 0.5 \\
\hline U-10 & 1.0 & 0.0 & 0.0 & 1.0 & 0.0 & 0.0 \\
\hline ICS-95 & 0.0 & 0.0 & 0.0 & 0.0 & 0.0 & 0.0 \\
\hline PORCELANA & 0.5 & 0.0 & 0.0 & 0.3 & 1.5 & 5.5 \\
\hline ROSARIO & 0.0 & 0.0 & 0.0 & 0.0 & 0.0 & 0.0 \\
\hline
\end{tabular}

\section{CONCLUSIONES}

Los clones promisorios de cacao destacan por su mayor productividad y adaptación a las condiciones ambientales, así como su moderada tolerancia a las principales plagas, son los clones ICS - 1, ICS - 60, ICS - 39, SCA - 6. Además, el clon TSH - 565 sobresale por la característica de su fino aroma. Estos deben ser recomendados para el cultivo de los productores, con la finalidad de reforzar las plantaciones y de esta manera elevar la productividad de plantas de cacao de pequeños, medianos productores $\mathrm{y}$ comunidades nativas, con la recomendación técnica de buen manejo agronómico a fin de mejorar su competitividad en el mercado local y nacional.

\section{Agradecimientos}

Los autores agradecen al Instituto Nacional de Innovación Agraria INIA.

\section{Referencias}

Álvarez, C., Liconte, N, Pereira, Y. C., Farías, A. G., Buscema, I., \& Láres, M. (2018). Perfil de calidad comercial del cacao venezolano (Theobroma cacao L.). Novum Scientarum, 3(7), 21-32.

Álvarez, J. R. (2018). Acumulación de metales pesados (Pb y Cd) en almendras de cacao durante el proceso de fermentación y secado. [tesis de mestría, Escola Superior de Turismo e Tecnología do Mar Instituto Politécnico de Leira]. https://iconline.ipleiria.pt/bitstream/10400.8/3478/1/ TESE-\%20Ricardo_final\%2029-062018_ENTREGAR.pdf

Ballesteros, W., Lagos, T. C., \& Ferney, H. (2016). Morphological characterization of elite cacao trees 
(Theobroma cacao L.) in Tumaco, Nariño, Colombia. Revista Colombiana de Ciencias Hortícolas, 9(2), 313328. http://dx.doi.org/10.17584/rcch.2015v9i2.4187

Food and Agriculture Organization (2002). Captura de carbono en los suelos para un mejor manejo de la tierra. Informe sobre recursos mundiales de suelos No. 96. Recuperado el 21 de junio de 2021, de http://books.google.es/books?hl=es\&lr=\&id=OKZt9a gfRksC\&oi=fnd\&pg=PR3\&dq=CAPTURA+DE+CAR $\mathrm{BONO}+\mathrm{EN}+\mathrm{LOS}+\mathrm{SUELOS}+\mathrm{PARA}+\mathrm{UN}+\mathrm{MEJOR}+\mathrm{MA}$ NEJO+DE+LA+TIERRA\&ots $=5 \times$ OjDqvtWf\&sig=c96h5Q4W_qU0xBHudD4IqchYyc

García, L. A. (2020). Caracterización morfológica en las zonas de producción de cacao (Theobroma cacao L.) tipo nacional en el cantón Babahoyo provincia de Los Ríos, Ecuador. [Tesis de grado, Universidad Técnica de Babahoyo]. http://dspace.utb.edu.ec/bitstream/handle/49000/849 3/E-UTB-FACIAG-ING\%20AGRON000283.pdf? sequence $=1 \&$ is Allowed $=y$

Garcia, P. V. (2010). Clasificacion Intraespecifica de 14 Arboles Hibridos Seleccionados de Cacao (Theobroma cacao L), mediante Analisis de Conglomerados en Tulumayo. [tesis de pregrado, Universidad Nacional Agraria de la Selva].

http://repositorio.unas.edu.pe/bitstream/handle/UN AS/90/AGR-534.pdf?sequence=1\&isAllowed=y

Gorotiza, J., Quevedo, J. N., \& García, R. M. (2020). Efectos del corte apical en semillas de cacao (Theobroma cacao L.) ICS 95 en sustratos con biocarbón para la obtención de portainjertos. Revista Científica AgroecoSistemas, 8(2): 66-72.

Ministerio de Agricultura y Riego. (2018). Análisis de la cadena productiva del cacao con enfoque en los pequeños productores de limitado acceso al mercado. Recuperado el 21 de junio de 2021, de https://repositorio.minagri.gob.pe/handle/MIDAGRI 166

Oliva, S. M. (2020). Caracterización socioeconómica de la diversidad biológica de cacao criollo fino de aroma en comunidades rurales de la región Amazonas. [tesis de posgrado, Universidad Nacional Toribio Rodríguez de Mendoza]. http://repositorio.untrm.edu.pe/bitstream/handle/U NTRM/2211/Oliva\%20Cruz\%20Segundo\%20Manuel $\% 20 \% 28$ cc $\% 29$.pdf? sequence=9\&isAllowed $=y$
Prado, F. (2019). Caracterización agronómica y morfológica de fruto y semilla de cuatro clones promisorios de cacao (Theobroma cacao L.), Kimbiri, Cusco. [Tesis de grado, Universidad Nacional de San Cristóbal de Huamanga].

http://repositorio.unsch.edu.pe/bitstream/handle/U NSCH/3602/TESIS\%20AF11_Pra.pdf?sequence=1\&is Allowed $=\mathrm{y}$

Quintana, L. F., Gómez, S., García, A., \& Martínez, N. (2015). Caracterización de tres índices de cosecha de cacao de los clones CCN51, ICS60 e ICS 95, en la montaña santandereana, Colombia. Revista de Investigación Agraria y Ambiental, 6 (1), 253-266.

Quiñones, M., Espinoza, E., Yovera, F., Cuchilla, Y., \& Castro, D. (2018). Identificación, georreferenciación y caracterización morfológica de árboles superiores de Theobroma Cacao L. 1753 cultivar cacao blanco de Piura, Perú. The Biologist, 16(1), 105-117. https://doi.org/10.24039/rtb2018161225

Ramírez-Guillermo, M. A., Lagunes-Espinoza, L. C., OrtizGarcía, C. F., Gutiérrez, O.A. \& de la RosaSantamaría, R. (2018). Variación morfológica de frutos y semillas de cacao (Theobroma cacao L.) de plantaciones de Tabasco, México. Revista Fitotecnia Mexicana, 41(2), 117-125.

Tarqui, O. M. (2020). Desempeño agroforestal y económico de los clones de cacao (Theobroma cacao L.) del CATIE en diferentes condiciones agroecológicas de Costa Rica. [Tesis de posgrado, Centro Agronómico Tropical de Investigación $\quad \mathrm{y} \quad$ Enseñanza]. http://repositorio.bibliotecaorton.catie.ac.cr/handle/1 1554/9842

Vera, J., Vallejo, C., Párraga, D., Morales, W., Macías, J., \& Ramos, R. (2014). Atributos físicos-químicos y sensoriales de las almendras de quince clones de cacao nacional (Theobroma cacao L.) en el Ecuador. Ciencia y Tecnología, 7(2), 21-34.

Xiao-Wei, Q, Jian-Xiong, L., Le-He, T., Chao-Yun, H., FuPeng, L., Shu-Zhen, H, \& Ying-Hui, S. (2017). Characterization of volatile compounds in Criollo, Forastero, and Trinitario cocoa seeds (Theobroma cacao L.) in China. International Journal of Food Properties, 20(10), 2261-2275. https://doi.org/10.1080/10942912.2016.1236270 